\title{
Fatores sociodemográficos e condições de risco em gestantes de um ambulatório especializado do sul do Brasil
}

\author{
Socio-demographic factors and risk conditions in pregnant women from a specialized \\ outpatient clinic in southern Brazil \\ Factores sociodemográficos y condiciones de riesgo en mujeres embarazadas de una \\ clínica ambulatoria especializada en el sur de Brasil
}

Marcos Benatti Antunes ${ }^{1,2 *}$, Angela Andréia França Gravena ${ }^{1}$, Geisa dos Santos Luz ${ }^{1}$, Leidyani Karina Rissardo ${ }^{1}$, Ludmila Lopes Maciel Bolsoni ${ }^{1}$, Patrícia Bossolani Charlo ${ }^{1}$, Thais Pordeus Leite Costa Mendes ${ }^{2}$, Marcela Demitto Furtado ${ }^{3}$, Sandra Marisa Pelloso ${ }^{3}$.

\section{RESUMO}

Objetivo: Analisar a presença de condições de riscos e sua relação com as características sociodemográficas de gestantes atendidas em um ambulatório especializado do sul do Brasil. Métodos: Estudo exploratório, descritivo, de abordagem quantitativa, realizado a partir de prontuários de 920 gestantes acompanhadas no pré-natal de alto risco. As variáveis desfechos foram: condições pré-existentes, antecedentes obstétricos e intercorrências clínicas e as variáveis independentes foram características sociodemográficas da mulher. Resultados: Mulheres com 36 anos ou mais apresentaram associação significativa para hipertensão $(28,8 \%)$, endocrinopatias $(20,6 \%)$, mioma uterino $(6,9 \%)$ e mais de três cesáreas $(6,9 \%)$. A baixa escolaridade esteve associada com hipertensão (25,0\%), epilepsia $(6,4 \%)$, mais de três cesáreas $(10,8 \%)$ e gemelaridade $(10,8 \%)$. Conclusão: Identificaram-se associações entre o perfil sociodemográfico com condições clínicas préexistentes, antecedentes obstétricos e intercorrências clínicas na gestação de alto risco, atribuindo aos ambulatórios especializados a necessidade de realizar ações para minimizar os desfechos desfavoráveis e melhorar a qualidade dos serviços.

Palavras-chave: Saúde da mulher, Complicações na gravidez, Ambulatório hospitalar.

\begin{abstract}
Objective: To analyze the presence of risk conditions and their relationship with the sociodemographic characteristics of pregnant women attending a specialized outpatient clinic in southern Brazil. Methods: An exploratory, descriptive study with a quantitative approach, conducted from medical records of 920 pregnant women followed at high risk prenatal care. The outcome variables were pre-existing conditions, obstetric history and clinical complications, and the independent variables were sociodemographic characteristics of women. Results: Women aged 36 years or older had a significant association with hypertension (28.8\%), endocrinopathies (20.6\%), uterine fibroid (6.9\%) and more than three cesarean sections (6.9\%). Low education was associated with hypertension (25.0\%), epilepsy (6.4\%), more than three cesarean sections (10.8\%) and twin pregnancy (10.8\%). Conclusion: Associations were identified between the sociodemographic profile with pre-existing clinical conditions, obstetric antecedents and clinical complications in high-risk pregnancy, attributing to specialized outpatient clinics the need to take actions to minimize unfavorable outcomes and improve the quality of services.
\end{abstract}

Key words: Women's health, Pregnancy complications, Outpatient clinic.

\section{RESUMEN}

Objetivo: Analizar la presencia de condiciones de riesgo y su relación con las características sociodemográficas de las mujeres embarazadas que acuden a una clínica ambulatoria especializada en el

\footnotetext{
${ }^{1}$ Centro Universitário de Maringá (UNICESUMAR), Maringá-Paraná. *E-mail: bena77i@gmail.com

2 Prefeitura do Município de Sarandi-PR.

3 Universidade Estadual de Maringá (UEM), Maringá-Paraná.
} 
sur de Brasil. Métodos: Un estudio exploratorio, descriptivo con un enfoque cuantitativo, realizado a partir de registros médicos de 920 mujeres embarazadas seguidas de atención prenatal de alto riesgo. Las variables de resultado fueron: afecciones preexistentes, antecedentes obstétricos y complicaciones clínicas, y las variables independientes fueron las características sociodemográficas de las mujeres. Resultados: Las mujeres de 36 años o más tuvieron una asociación significativa con hipertensión (28.8\%), endocrinopatías (20.6\%), fibromas uterinos $(6.9 \%)$ y más de tres cesáreas $(6.9 \%)$. La baja educación se asoció con hipertensión (25.0\%), epilepsia (6.4\%), más de tres cesáreas (10.8\%) y embarazo gemelar (10.8\%). Conclusión: Se identificaron asociaciones entre el perfil sociodemográfico con condiciones clínicas preexistentes, antecedentes obstétricos y complicaciones clínicas en embarazos de alto riesgo, atribuyendo a clínicas ambulatorias especializadas la necesidad de tomar medidas para minimizar los resultados desfavorables y mejorar la calidad de los servicios.

Palabras clave: Salud de la mujer, Complicaciones del embarazo, Consulta externa.

\section{INTRODUÇÃO}

A mortalidade materna em todo mundo caiu 45\% entre 1990 e 2013, porém, cerca de 9.300 mulheres ainda morrem anualmente por essa causa. Para a Organização Mundial de Saúde (OMS), mais de uma em cada quatro mortes maternas são por condições pré-existentes, como diabetes, Vírus da Imunodeficiência Humana (HIV), malária, obesidade e que podem ser agravadas na gravidez. Esses números são semelhantes aos óbitos por hemorragia grave durante a gestação e o parto (WHO, 2014).

Apesar da diminuição no índice de mortalidade por complicações na gravidez, parto e puerpério, é preciso ainda atenção, uma vez que os países em desenvolvimento não foram capazes de alcançar o Objetivo de Desenvolvimento do Milênio (ODM) de reduzir a mortalidade materna em 75\% até 2015 (WHO, 2014).

Com o objetivo de reduzir a morbimortalidade materno-infantil no Brasil, o Ministério da Saúde (MS) instituiu, pela portaria $n^{\circ} 1.459$ de 2011, a Rede Cegonha. Este programa propõe melhor atendimento às mulheres durante a gestação, parto e puerpério, assim como ao recém-nascido e às crianças até os dois anos de idade. Objetiva também qualificar as redes de atenção materno-infantis, garantindo acesso, acolhimento e resolutividade das ações (MINISTÉRIO DA SAÚDE - BRASIL, 2011).

No Estado do Paraná foi criada a Rede Mãe Paranaense (RMP), que se apresenta como um conjunto de ações que envolvem desde a aproximação precoce com as gestantes, acompanhamento no pré-natal na atenção primária até, quando em situação de risco, o acompanhamento na atenção secundária em ambulatórios de risco, garantindo também o parto e consultas de emergência na atenção terciária (SECRETARIA ESTADUAL DE SAÚDE - PARANÁ, 2018).

A RMP prevê ações de atenção primária nos 399 municípios do Paraná e, nas atenções secundária e terciária, a implantação de ambulatórios para o atendimento de gestante e criança de risco nas 22 regiões de saúde do estado (SECRETARIA ESTADUAL DE SAÚDE - PARANÁ, 2018).

A presença de fatores de risco associada à gestação pode contribuir com o desencadeamento de patologias. Na maioria das vezes, uma gestação evolui de maneira natural, sem intercorrências clínicas. Entretanto, quando a mulher apresenta doenças pregressas ou algumas intercorrências neste período, a gestação se torna de risco, com evolução desfavorável, expondo a vida da mãe e/ou do feto a agravos (BRASIL, MINISTÉRIO DA SAÚDE, 2012a).

Algumas pesquisas discutem o aumento de comorbidades relacionadas com a gravidez (GONÇALVES ZR e MONTEIRO DLM, 2012; FRIDMAN M, et al., 2014), porém ainda faltam estudos que avaliam a associação entre os fatores sociodemográficos e todas as condições pré-clínicas e clínicas de gestantes de risco descritas pelo Ministério da Saúde (MS), o que justifica esse estudo e, além disso, seus resultados poderão auxiliar os ambulatórios da RMP na implementação e melhoria da assistência ao pré-natal de risco, contribuindo com a redução das taxas de morbimortalidade materna e infantil. Desse modo, o objetivo foi analisar a presença de condições de risco e sua relação com as características sociodemográficas de gestantes atendidas em um ambulatório especializado do Sul do Brasil. 


\section{MÉTODOS}

Estudo exploratório, retrospectivo, descritivo, com base documental e de abordagem quantitativa, realizado com gestantes atendidas em consultas de pré-natal de risco em um ambulatório de um hospital de um hospital do estado do Paraná, Brasil.

A população foi representada por todas as mulheres atendidas e classificadas como alto risco, no período de setembro de 2012 a setembro de 2013, configurando um ano de atendimento no ambulatório. Os dados foram coletados de novembro de 2016 a outubro de 2017 a partir dos relatórios emitidos a partir dos prontuários das gestantes e score de classificação de risco utilizado pela atenção primária de saúde, para encaminhamento da gestante ao ambulatório (atenção secundária), este documento contém a relação de condições clínicas pré-existentes, antecedentes obstétricos e intecorrências clínicas da atual gestação, que a classifica como alto risco. As informações foram registradas em um formulário previamente elaborado, denominado Gestantes de Alto Risco (GAR) e inserido em planilha eletrônica da Microsoft Corporation Redmond, WA, USA.

As variáveis independentes referentes à mulher foram: idade, raça, estado civil, escolaridade e religião. As variáveis 'desfechos' foram as condições de risco gestacional, apresentadas pelas gestantes no score, as quais foram classificadas em três grupos: Condição clínica pré-existente, composto por hipertensão arterial (leve, moderada e grave), cardiopatias (doença valvar cardíaca, doença de chagas, cardiopatias congênitas e cardiopatias isquêmicas), pneumopatias (asma, doença pulmonar obstrutiva crônica e embolia pulmonar), nefropatias (insuficiência renal crônica e insuficiência renal aguda), endocrinopatias (diabetes controlada por dieta, diabetes com uso de insulina, hipotireoidismo e hipertireoidismo), hemopatias (talassemia, anemia falciforme e púrpura trombocitopenêmica), doenças autoimunes (trombose venosa profunda, trombofilias, lúpus eritematoso sistêmico), doenças infecciosas (infecção HIV/aids, hepatites B e C, toxoplasmose, tuberculose e hanseníase), epilepsia, conização, mioma uterino, neoplasias, obesidade mórbida, cirurgia bariátrica, psicose e depressão grave e drogas lícitas e ilícitas.

Antecedentes obstétricos, composto por mortes fetais e neonatais, baixo peso ao nascer $(<2.500 \mathrm{~g})$, mais de três cesáreas, acretismo placentário, hemorragia de segundo semestre (placenta prévia ou descolamento prematuro de placenta), pré-eclâmpsia, cerclagem anterior, amniorrexe prematura, trabalho de parto prétermo, parto pré-termo e aborto de repetição. Intercorrências clínicas, compostas por infecção do trato urinário (complicada, de repetição e pielonefrite), rubéola, toxoplasmose, pré-eclâmpsia, retardo do crescimento intrauterino, trabalho de parto pré-termo, placenta prévia, aminiorrexe prematura ( $<$ de 37 semanas), isoimunização $\mathrm{RhD}$, malformação fetal confirmada, macrossomia (do concepto com patologias) e gemelaridade.

Para a compilação dos dados e análise estatística foi utilizado o software Epi Info, versão 3.5.1, programa de domínio público criado pelo Centers for Disease Control and Prevention (CDC). Os dados foram submetidos aos testes Qui-quadrado $\left(\mathrm{x}^{2}\right)$ e Teste Exato de Fisher. Todas as discussões foram realizadas em nível de $5 \%$ de significância $(p<0,05)$ e intervalo de confiança de $95 \%$.

$O$ desenvolvimento do estudo atendeu as normas nacionais e internacionais de ética em pesquisa envolvendo seres humanos, sendo aprovado pelo parecer oㅜ 2.287.476 do Comitê Permanente de Ética em Pesquisa com Seres Humanos da Universidade Estadual de Maringá (COPEP).

\section{RESULTADOS}

A amostra contou com 920 gestantes classificadas como alto risco e acompanhadas no ambulatório especializado. A população estudada apresentou média de $28,34( \pm 6,82)$ anos (idade mínima de 12 e máxima de 47 anos) e 698 (75,9\%) das mulheres concentraram-se na faixa etária de 19-35 anos, seguindo de 160 $(17,4 \%)$ com idade de 36 anos ou mais. A maioria das mulheres $549(60,1 \%)$ era casada ou viviam em união estável, $342(37,4 \%)$ eram solteiras e ainda $5(0,5 \%)$ eram gestantes viúvas. Em relação à escolaridade, 716 $(77,8 \%)$ estudaram mais de oito anos. Quanto à religião, $564(67,20 \%)$ eram católicas e $265(28,8 \%)$ evangélicas; 580 (63,04\%) declararam-se brancas (Tabela 1). 
Tabela 1 - Distribuição das variáveis sociodemográficas das gestantes acompanhadas em ambulatório de risco, n=920. Maringá, PR, Brasil, 2017.

\begin{tabular}{|c|c|c|}
\hline Variáveis & $\mathrm{n}$ & $\%$ \\
\hline \multicolumn{3}{|l|}{ Idade } \\
\hline 10 a 18 & 62 & 6,7 \\
\hline 19 a 35 & 698 & 75,9 \\
\hline 36 ou mais & 160 & 17,4 \\
\hline \multicolumn{3}{|l|}{ Cor } \\
\hline Branca & 580 & 63,0 \\
\hline Preta & 43 & 4,7 \\
\hline Parda/morena & 296 & 32,2 \\
\hline Amarela & 1 & 0,1 \\
\hline \multicolumn{3}{|l|}{ Estado civil } \\
\hline Casada/união estável & 549 & 60,1 \\
\hline Solteira & 342 & 37,4 \\
\hline Separada/divorciada & 18 & 2,0 \\
\hline Viúva & 5 & 0,5 \\
\hline \multicolumn{3}{|l|}{ Escolaridade } \\
\hline$<8$ anos & 204 & 22,2 \\
\hline$\geq 8$ anos & 716 & 77,8 \\
\hline \multicolumn{3}{|l|}{ Religião } \\
\hline Católica & 564 & 67,2 \\
\hline Evangélica & 265 & 31,6 \\
\hline Sem religião & 10 & 1,2 \\
\hline
\end{tabular}

Fonte: Antunes MB, et al., 2019

A análise das condições clínicas pré-existentes das gestantes de acordo com as variáveis sociodemográficas está apresentada na Tabela 2. Gestantes com idade de 36 anos ou mais apresentaram frequência superior de doenças hipertensivas, endocrinopatias e mioma uterino.

A epilepsia esteve presente entre aquelas com idade entre dez e 18 anos. Em relação ao estado civil, sete $(38,9 \%)$ das gestantes separadas/divorciadas apresentaram doenças hipertensivas, três $(16,7 \%)$ obesidade mórbida e dois $(11,1 \%)$ realizaram cirurgia bariátrica.

Entre as solteiras, $33(9,6 \%)$ apresentaram dependência de drogas lícitas/ilícitas e três $(60,0 \%)$ das gestantes viúvas apresentaram endocrinopatias. Quanto à escolaridade, mulheres com $<8$ anos de estudo foram caracterizadas em sua maioria com hipertensão arterial e epilepsia. Um total de 10,0\% das gestantes que não apresentaram religião foi caracterizado com presença de neoplasias.

A Tabela 3, mostra a associação entre antecedentes obstétricos e variáveis sociodemográficas. Mulheres com idade de 36 anos ou mais apresentaram valores significativos para mais de três cesáreas com $11(6,9 \%)$ das mulheres e aborto de repetição 17 (10,6\%). A pré-eclâmpsia esteve presente entre as gestantes de 19 a 35 anos. Em relação à cor, gestantes que se declararam de cor parda/morena apresentaram maior frequência de mortes fetais e neonatais. Uma única gestante que se declarou de cor amarela apresentou $\mathrm{BPN}<1.500 \mathrm{~g}$ e hemorragia do segundo semestre. Gestantes com estado civil separada/divorciada possuíram mais de três cesáreas e as viúvas apresentaram pré-eclâmpsia.

As intercorrências clínicas e sua associação com as variáveis sociodemográficas demonstraram que gestantes com idade de dez a 18 anos apresentaram Retardo de Crescimento Intrauterino (RCIU), trabalho de parto pré-termo e malformação fetal intra útero. Em relação à escolaridade, $22(10,8 \%)$ daquelas com $<8$ anos de estudos tiveram gestação gemelar. Quanto à religião, as mulheres sem religião apresentaram toxoplasmose e amniorrexe prematura (Tabela 4). 


\section{Revista Eletrônica Acervo Saúde / Electronic Journal Collection Health ｜ ISSN 2178-2091}

Tabela 2 - Condições clínicas pré-existentes ${ }^{\S}$ das gestantes de alto risco segundo variáveis sociodemográficas. Maringá, PR, Brasil, 2017.

\begin{tabular}{|c|c|c|c|c|c|c|c|c|c|c|c|c|c|c|c|c|}
\hline Variáveis & $\begin{array}{l}\text { HAS } \\
\mathrm{n}(\%)\end{array}$ & $\mathrm{p}$ & $\begin{array}{c}\text { Endocrino } \\
\text { patias } \\
\mathrm{n}(\%)\end{array}$ & $p$ & $\begin{array}{c}\text { Epilepsia } \\
\mathrm{n}(\%)\end{array}$ & $p$ & $\begin{array}{c}\text { Mioma } \\
\text { Uterino } \\
\mathrm{n}(\%)\end{array}$ & $p$ & $\begin{array}{c}\text { Neoplasias } \\
\text { n (\%) }\end{array}$ & $\mathrm{p}$ & $\begin{array}{c}\text { Obesidade } \\
\text { mórbida } \\
\mathrm{n}(\%)\end{array}$ & $\mathrm{p}$ & $\begin{array}{c}\text { Cirurgia } \\
\text { Bariátrica } \\
\mathrm{n}(\%)\end{array}$ & $p$ & $\begin{array}{l}\text { Dep. drogas } \\
\text { lícitas/ } \\
\text { ilícitas } \\
\mathrm{n}(\%) \\
\end{array}$ & $p$ \\
\hline Idade & & $<0,001^{*}$ & & $0,006^{*}$ & & $0,038^{*}$ & & $0,003^{*}$ & & $0,651^{*}$ & & $0,083^{*}$ & & $0,136^{*}$ & & $0,921^{*}$ \\
\hline 10 a 18 & $5(8,1)$ & & $2(3,2)$ & & $3(4,8)$ & & - & & $1(1,6)$ & & $1(1,6)$ & & - & & $4(6,5)$ & \\
\hline 19 a 35 & $114(16,3)$ & & $114(16,3)$ & & $26(3,7)$ & & $16(2,3)$ & & $5(0,7)$ & & $42(6,0)$ & & $6(0,9)$ & & $39(5,6)$ & \\
\hline 36 ou mais & $46(28,8)$ & & $33(20,6)$ & & - & & $11(6,9)$ & & $2(1,3)$ & & $15(9,4)$ & & $4(2,5)$ & & $10(6,3)$ & \\
\hline Cor & & $0,064^{*}$ & & $0,111^{*}$ & & $0,153^{*}$ & & $0,674^{*}$ & & $0,928^{*}$ & & $0,513^{*}$ & & $0,703^{*}$ & & $0,829^{*}$ \\
\hline$\overline{\text { Branca }}$ & $109(18,8)$ & & $91(15,7)$ & & $13(2,2)$ & & $17(2,9)$ & & $5(0,9)$ & & $36(6,2)$ & & $8(1,4)$ & & $31(5,3)$ & \\
\hline Preta & $13(30,2)$ & & $9(20,9)$ & & $1(2,3)$ & & - & & - & & $5(11,6)$ & & - & & $2(4,7)$ & \\
\hline Parda & $43(14,5)$ & & $48(16,2)$ & & $15(5,1)$ & & $10(3,4)$ & & $3(1,0)$ & & $17(5,7)$ & & $2(0,7)$ & & $20(6,8)$ & \\
\hline Amarela & - & & $1(100,0)$ & & - & & - & & - & & - & & - & & - & \\
\hline Estado civil & & $0,042^{*}$ & & $0,007^{*}$ & & $0,303^{*}$ & & $0,185^{\star}$ & & $0,193^{*}$ & & $0,036^{*}$ & & $<0,001^{*}$ & & $0,001^{*}$ \\
\hline Casada & $106(19,3)$ & & $92(16,8)$ & & $13(2,4)$ & & $17(3,1)$ & & $4(0,7)$ & & $40(7,3)$ & & $6(1,1)$ & & $18(3,3)$ & \\
\hline Solteira & $51(14,9)$ & & $44(12,9)$ & & $15(4,4)$ & & $8(2,3)$ & & $3(0,9)$ & & $14(4,1)$ & & $2(0,6)$ & & $33(9,6)$ & \\
\hline Divorciada & $7(38,9)$ & & $7(38,9)$ & & - & & $2(11,1)$ & & $1(5,6)$ & & $3(16,7)$ & & $2(11,1)$ & & $1(5,6)$ & \\
\hline Viúva & $1(20,0)$ & & $3(60,0)$ & & - & & - & & 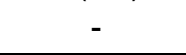 & & $1(20,0)$ & & - & & & \\
\hline Escolaridade & & $0,002^{*}$ & & $0,822^{*}$ & & $0,002^{*}$ & & $0,249^{\star \star}$ & & $0,440^{*}$ & & $0,709^{*}$ & & $0,080^{\star \star}$ & & $0,073^{*}$ \\
\hline$<8$ anos & $51(25,0)$ & & $32(15,7)$ & & $13(6,4)$ & & $4(2,0)$ & & $1(0,5)$ & & $14(6,9)$ & & - & & $17(8,3)$ & \\
\hline$\geq 8$ anos & $114(15,9)$ & & $117(16,3)$ & & $16(2,2)$ & & $23(3,2)$ & & $7(1,0)$ & & $44(6,1)$ & & $10(1,4)$ & & $36(5,0)$ & \\
\hline Religião & & $0,397^{\star}$ & & $0,609^{*}$ & & $0,856^{*}$ & & $0,307^{\star}$ & & $0,010^{*}$ & & $0,371^{*}$ & & $0,774^{*}$ & & $0,150^{*}$ \\
\hline Católica & $98(17,4)$ & & $87(15,4)$ & & $16(2,8)$ & & $15(2,7)$ & & $4(0,7)$ & & $33(5,9)$ & & $7(1,2)$ & & $37(6,6)$ & \\
\hline Evangélica & $55(20,8)$ & & $47(17,7)$ & & $7(2,6)$ & & $12(4,5)$ & & $3(1,1)$ & & $21(7,9)$ & & $2(0,8)$ & & $9(3,4)$ & \\
\hline Sem religião & $1(10,0)$ & & $1(1,0)$ & & - & & - & & $1(10,0)$ & & - & & - & & $1(10,0)$ & \\
\hline
\end{tabular}

Legenda: §As condições clínicas: cardiopatias, pneumopatias, nefropatias, hemopatias, doenças autoimunes, doenças infecciosas, conização, psicose e depressão grave não apresentaram associação significativa com as variáveis. * $p$-valor obtido por meio do teste Qui-quadrado; ** p-valor obtido por meio do teste exato de Fisher. Fonte: Antunes MB, et al., 2019. 


\section{Revista Eletrônica Acervo Saúde / Electronic Journal Collection Health ISSN 2178-2091}

Tabela 3 - Antecedentes obstétricos§ das gestantes de alto risco segundo variáveis sociodemográficas. Maringá, PR, Brasil, 2017.

\begin{tabular}{|c|c|c|c|c|c|c|c|c|c|c|c|c|c|c|}
\hline Variáveis & $\begin{array}{c}\text { Morte fetais } \\
\text { e neonatais } \\
n(\%)\end{array}$ & $p$ & $\begin{aligned} & \mathrm{BPN} \\
&< 1500 \mathrm{~g} \\
& \mathrm{n}(\%)\end{aligned}$ & $p$ & $\begin{array}{c}\text { Mais de } \\
03 \\
\text { cesáreas } \\
\text { n (\%) }\end{array}$ & $p$ & $\begin{array}{c}\text { Hemorragia } \\
\text { do } 2^{\circ} \\
\text { semestre } \\
\text { n (\%) }\end{array}$ & $p$ & $\begin{array}{c}\text { Pré- } \\
\text { eclampsia } \\
\text { n (\%) }\end{array}$ & $p$ & $\begin{array}{c}\text { Amniorrexe } \\
\text { prematura } \\
\mathrm{n}(\%)\end{array}$ & $p$ & $\begin{array}{c}\text { Aborto de } \\
\text { repetição } \\
\text { n (\%) }\end{array}$ & $p$ \\
\hline Idade & & $0,202^{*}$ & & $0,364^{*}$ & & $0,006^{*}$ & & $0,575^{*}$ & & $0,041^{*}$ & & $0,262^{*}$ & & $0,022^{*}$ \\
\hline 10 a 18 & $2(3,2)$ & & $2(3,2)$ & & - & & $1(1,6)$ & & - & & - & & - & \\
\hline 19 a 35 & $61(8,7)$ & & $42(6,0)$ & & $18(2,6)$ & & $28(4,0)$ & & $62(8,9)$ & & $12(1,7)$ & & $54(7,7)$ & \\
\hline 36 ou mais & $10(6,3)$ & & $13(8,1)$ & & $11(6,9)$ & & $5(3,1)$ & & $12(7,3)$ & & $5(3,1)$ & & $17(10,6)$ & \\
\hline Cor & & $0,010^{*}$ & & $0,001^{*}$ & & $0,621^{*}$ & & $<0,001^{*}$ & & $0,447^{*}$ & & $0,082^{*}$ & & $0,225^{*}$ \\
\hline Branca & $39(6,7)$ & & $36(6,2)$ & & $15(2,6)$ & & $22(3,8)$ & & $41(7,1)$ & & $9(1,6)$ & & $38(6,6)$ & \\
\hline Preta & - & & $2(4,7)$ & & $2(4,7)$ & & - & & $3(7,0)$ & & $3(7,0)$ & & $6(14,0)$ & \\
\hline Parda & $34(11,5)$ & & $18(6,1)$ & & $12(4,1)$ & & $11(3,7)$ & & $30(10,1)$ & & $5(1,7)$ & & $27(9,1)$ & \\
\hline Amarela & - & & $1(100,0)$ & & - & & $1(100,0)$ & & - & & - & & - & \\
\hline Estado civil & & $0,546^{*}$ & & $0,082^{*}$ & & $<0,001^{*}$ & & $0,415^{\star}$ & & $0,019^{*}$ & & $0,614^{*}$ & & $0,146^{*}$ \\
\hline Casada & $39(7,1)$ & & $26(4,7)$ & & $15(2,7)$ & & $16(2,9)$ & & $38(6,9)$ & & $11(2,0)$ & & $51(9,3)$ & \\
\hline Solteira & $32(9,4)$ & & $29(8,5)$ & & $7(2,0)$ & & $17(5,0)$ & & $30(8,8)$ & & $5(1,5)$ & & $18(5,3)$ & \\
\hline Ddivorciada & $2(11,1)$ & & $1(5,6)$ & & $5(27,8)$ & & $1(5,6)$ & & $3(16,7)$ & & $1(5,6)$ & & $1(5,6)$ & \\
\hline Viúva & - & & $1(20,0)$ & & $1(20,0)$ & & - & & $2(40,0)$ & & - & & - & \\
\hline Escolaridade & & $0,087^{*}$ & & $0,002^{*}$ & & $0,104^{*}$ & & $0,846^{*}$ & & $0,295^{\star}$ & & $0,182^{*}$ & & $0,331^{*}$ \\
\hline$<8$ anos & $22(10,8)$ & & $22(10,8)$ & & $10(4,9)$ & & $8(3,9)$ & & $20(9,8)$ & & $6(2,9)$ & & $19(9,3)$ & \\
\hline$\geq 8$ anos & $51(7,1)$ & & $35(4,9)$ & & $19(2,7)$ & & $26(3,6)$ & & $54(7,5)$ & & $11(1,5)$ & & $52(7,3)$ & \\
\hline Religião & & $0,542^{*}$ & & $0,161^{*}$ & & $0,771^{*}$ & & $0,009^{*}$ & & $0,503^{*}$ & & $0,022^{\star}$ & & $0,483^{*}$ \\
\hline Católica & $47(8,3)$ & & $32(5,7)$ & & $16(2,8)$ & & $16(2,8)$ & & $43(7,6)$ & & $6(1,1)$ & & $40(7,1)$ & \\
\hline Evangélica & $19(7,2)$ & & $16(6,0)$ & & $9(3,4)$ & & $12(4,5)$ & & $24(9,1)$ & & $10(3,8)$ & & $25(9,4)$ & \\
\hline Sem religião & - & & $2(20,0)$ & & - & & $2(20,0)$ & & - & & - & & $1(10,0)$ & \\
\hline
\end{tabular}

Legenda: §Os antecedentes obstétricos: acretismo, cerclagem anterior, trabalho de parto pré-termo e parto pré-termo não apresentaram associação significativa com as variáveis. * p-valor obtido por meio do teste Qui-quadrado; ${ }^{* *} p$-valor obtido por meio do teste exato de Fisher.

Fonte: Antunes MB, et al., 2019. 


\section{Revista Eletrônica Acervo Saúde / Electronic Journal Collection Health ｜ ISSN 2178-2091}

Tabela 4 - Intercorrências clínicas§ das gestantes de alto risco segundo variáveis sociodemograficas. Maringá, PR, Brasil, 2017.

\begin{tabular}{|c|c|c|c|c|c|c|c|c|c|c|c|c|}
\hline Variáveis & $\begin{array}{c}\text { Toxoplasmose } \\
\text { n (\%) }\end{array}$ & $\mathrm{p}$ & $\begin{array}{c}\text { Retardo de } \\
\text { crescimento } \\
\text { intrauterino } \\
\mathrm{n}(\%)\end{array}$ & $p$ & $\begin{array}{c}\text { Trabalho } \\
\text { de parto } \\
\text { pré-termo } \\
\mathrm{n}(\%)\end{array}$ & $p$ & $\begin{array}{c}\text { Aminiorrexe } \\
\text { prematura } \\
\mathrm{n}(\%)\end{array}$ & $p$ & $\begin{array}{c}\text { Malformação } \\
\text { Fetal intra útero } \\
\mathrm{n}(\%)\end{array}$ & $p$ & $\begin{array}{c}\text { Gestações } \\
\text { gemelares } \\
\mathrm{n}(\%)\end{array}$ & $p$ \\
\hline Idade & & $0,382^{*}$ & & $0,010^{*}$ & & $0,035^{*}$ & & $0,791^{*}$ & & $<0,001^{*}$ & & $0,421^{*}$ \\
\hline 10 a 18 & - & & $4(6,5)$ & & $6(9,7)$ & & $1(1,6)$ & & $8(12,9)$ & & $3(4,8)$ & \\
\hline 19 a 35 & $6(0,9)$ & & $10(1,4)$ & & $25(3,6)$ & & $7(1,0)$ & & $29(4,2)$ & & $56(8,0)$ & \\
\hline 36 ou mais & - & & $2(1,3)$ & & $4(2,5)$ & & $1(0,6)$ & & $2(1,3)$ & & $9(5,6)$ & \\
\hline Cor & & $0,502^{*}$ & & $0,816^{*}$ & & $0,755^{*}$ & & $0,792^{*}$ & & $0,638^{*}$ & & $0,263^{*}$ \\
\hline Branca & $4(0,7)$ & & $10(1,7)$ & & $25(4,3)$ & & $7(1,2)$ & & $22(3,8)$ & & $43(7,4)$ & \\
\hline Preta & $1(2,3)$ & & - & & $1(2,3)$ & & - & & $1(2,3)$ & & - & \\
\hline Parda & $1(0,3)$ & & $6(2,0)$ & & $9(3,0)$ & & $2(0,7)$ & & $16(5,4)$ & & $25(8,4)$ & \\
\hline Amarela & - & & - & & - & & - & & - & & - & \\
\hline Estado civil & & $0,701^{*}$ & & $0,446^{*}$ & & $0,391^{*}$ & & $0,127^{*}$ & & $0,755^{*}$ & & $0,548^{*}$ \\
\hline Casada & $5(0,9)$ & & $7(1,3)$ & & $17(3,1)$ & & $3(0,5)$ & & $23(4,2)$ & & $40(7,3)$ & \\
\hline Solteira & $1(0,3)$ & & $9(2,6)$ & & $17(5,0)$ & & $3(1,5)$ & & $16(4,7)$ & & $28(8,2)$ & \\
\hline Divorciada & - & & - & & - & & $1(5,6)$ & & - & & - & \\
\hline Viúva & - & & - & & - & & - & & - & & - & \\
\hline Escolaridade & & $0,601^{* *}$ & & $0,783^{*}$ & & $0,920^{*}$ & & $0,372^{\star \star}$ & & $0,594^{*}$ & & $0,035^{*}$ \\
\hline$<8$ anos & $1(0,5)$ & & $4(2,0)$ & & $8(3,9)$ & & $1(0,5)$ & & $10(4,9)$ & & $22(10,8)$ & \\
\hline$\geq 8$ anos & $5(0,7)$ & & $12(1,7)$ & & $27(3,8)$ & & $8(1,1)$ & & $29(4,1)$ & & $46(6,4)$ & \\
\hline Religião & & $<0,001^{*}$ & & $0,919^{*}$ & & $0,248^{*}$ & & $0,007^{*}$ & & $0,568^{*}$ & & $0,167^{*}$ \\
\hline Católica & $3(0,5)$ & & $9(1,6)$ & & $15(2,7)$ & & $6(1,1)$ & & $27(4,8)$ & & $51(9,0)$ & \\
\hline Evangélica & $1(0,4)$ & & $4(1,5)$ & & $11(4,2)$ & & $1(0,4)$ & & $10(3,8)$ & & $14(5,3)$ & \\
\hline Sem religião & $1(1,0)$ & & - & & $1(10,0)$ & & $1(10,0)$ & & $1(10,0)$ & & $1(10,0)$ & \\
\hline
\end{tabular}

Legenda: §As intercorrências clínicas: ITU complicada, rubéola, pré-eclâmpsia, placenta prévia, isoimunização RhD e macrossomia não apresentaram associação significativa com as variáveis. * $p$-valor obtido por meio do teste Qui-quadrado; ** p-valor obtido por meio do teste exato de Fisher.

Fonte: Antunes MB, et al., 2019. 


\section{DISCUSSÃO}

Mediante os resultados encontrados nesta pesquisa, constatou-se que as doenças hipertensivas, endocrinopatias e mioma uterino são as condições pré-existentes mais frequentes em gestantes de risco com idade de 36 anos ou mais. Outra análise retratou que essa relação acerca de um maior risco obstétrico em gestantes com idade avançada é decorrente da frequência aumentada de doenças crônicas, hipertensão, diabetes mellitus e miomas que acarretam em riscos potenciais na gestação (LISONKOVA S, PARÉ E e JOSEPH KS, 2013), entretanto, vale destacar que o mioma uterino muitas vezes se constitui em uma condição benigna que acomete cerca de 20 a 30\% das mulheres em idade fértil ( $>30$ anos) e pode estar presente em 2 a $3 \%$ das gestações e em cerca de $10 \%$ dos casos podem apresentar algum tipo de complicação no período gestacional e/ou puerperal (LEITE GKC, et al., 2010).

Outro resultado encontrado nesta pesquisa foi de que mulheres com idade avançada possuem mais de três cesáreas em seu histórico de antecedente obstétrico. Coerente a isso, um estudo realizado com 2.441 prontuários de partos assistidos, constatou que mulheres submetidas à cesariana na atual gestação passaram pelo mesmo procedimento em gestações anteriores (Razão de Prevalência - RP $=3,19$; Intervalo de Confiança - IC95\%: 2,64; 3,84) (OSAVA RH, et al., 2011). Vale destacar ainda que, quanto maior a idade da mulher, maior a taxa de cesariana; sendo assim, mulheres com 35 anos ou mais apresentam o dobro de prevalência desta intervenção do que aquelas com menos de 20 anos; sendo que as mulheres com idade maior que 44 anos estão associadas a um risco de até quatro vezes mais (GONÇALVES ZR e MONTEIRO DLM, 2012; PÁDUA KS, et al., 2010).

Nesse contexto, políticas públicas em relação ao parto cesáreo precisam ser revistas no cenário brasileiro, a fim de reduzir as taxas entre $10 \%$ e $15 \%$ desta intervenção, conforme recomendação da OMS, observando as indicações para tal procedimento, mesmo em gestação de alto risco (OMS, 2015).

$\mathrm{Na}$ faixa etária de mulheres com 36 anos ou mais, identificou-se a prevalência de abortos de repetição em seus antecedentes obstétricos, dados que estão de acordo com a literatura recente, os quais apontam que 0 risco de abortamento aumenta com a paridade, bem como com a idade materna. Em mulheres acima de 40 anos, esse fator potencializa em aproximadamente 26\% (MINISTÉRIO DA SAÚDE - BRASIL, 2012b).

A gravidez em idade superior a 35 anos é um fator de risco gestacional pré-existente que está aumentando mundialmente, exigindo atenção especial dos profissionais quanto ao seu seguimento, com o objetivo de minimizar eventos adversos comuns nessa faixa etária como: hipertensão, diabetes gestacional, maior frequência de cesariana em trabalho de parto pré-termo, placenta prévia, amniorrexe prematura e gestações múltiplas, 5 incluindo os desfechos desfavoráveis como a mortalidade materna, fetal e infantil.

Entre as gestantes de 19 a 35 anos, a pré-eclâmpsia foi o antecedente obstétrico prevalente. Outra pesquisa corrobora com valores significativos, ao realizar o levantamento de 250 prontuários de mulheres com pré-eclâmpsica e identificar que a maioria $(63,2 \%)$ estava inserida na faixa etária de 20 a 34 anos e 27,2\% apresentaram antecedentes clínicos de hipertensão/pré-eclâmpsia (LINHARES JJ, et al., 2014).

O resultado encontrado de prevalência da pré-eclâmpsia como antecedente obstétrico indica que essa recorrência está presente em gestantes em idade fértil, devendo os profissionais da Atenção Primária da Saúde (APS) atentarem à estratificação de risco nas consultas de pré-natal e visitas domiciliares. Essa medida identifica precocemente as gestantes que já passaram pela experiência de vivenciarem uma gravidez de risco e encaminharem à atenção secundária para atendimento especializado.

Com relação às mulheres com idade entre dez e 18 anos, constataram-se dados significativos para a epilepsia $(4,8 \%)$ como condições clínicas pré-existentes, resultado este acima da ocorrência na gravidez $(0,3$ a $0,8 \%$ ), por se tratar de uma doença neurológica rara entre mulheres grávidas, com risco aumentado de indução ao parto, cesariana e hemorragia pós-parto (BROSH K, et al., 2011). Embora faltem estudos sobre a prevalência de epilepsia na gestação em adolescentes e considerando que este distúrbio neorológico é o mais freqüente em obstetrícia, estima-se que $25 \%$ das mulheres com epilepsia estão em idade fértil (LUNARDI LL, et al., 2011). 
Neste sentido, essas gestantes devem ser acompanhadas com atenção específica a medicação de uso contínuo na gravidez. As drogas antiepilépticas podem ocasionar outros riscos à gestação, como o aborto, parto pré-termo e sangramento vaginal, sendo o melhor tratamento durante a gravidez a monoterapia com a menor dose possível (NIJAFI MR, et al., 2012).

A malformação fetal neste estudo, também se destacou entre as gestantes de dez a 18 anos. Nessa perspectiva, um estudo realizado com 174 prontuários com casos de malformação congênita identificou o predomínio de mães com 21 a 30 anos (44,8\%), seguido de mães com 10 a 20 anos (43,1\%) (REIS LLAS e FERRARI R, 2014). Em outro estudo, realizado em São Paulo, com 6257 gestações, das quais 577 (9,2\%) resultaram em recém-nascidos com anomalia congênita, 14,5\% tinham idade inferior a 20 anos, das quais $9,9 \%$ apresentaram anomalias que quando comparadas com mulheres com idade superior a 20 anos, apresentaram diferença estatística significativa apenas para defeitos do tubo neural $(p=0,027)$ (REIS LV, et al., 2015).

Com relação ao Retardo do Crescimento Intrauterino (RCIU) e o trabalho de parto prematuro foram intercorrências clínicas que apresentaram significância entre adolescentes na faixa etária de dez a 18 anos. Outro estudo que se propôs a analisar 335.590 mães no período de 2002 a 2011, em Taiwan, constatou que as mães adolescentes de 16-19 anos e as $\leq 15$ anos, sobretudo aquelas com idade entre 10-15 anos, apresentaram risco significativamente maior de RCIU (OR = 1,37, $95 \mathrm{Cl} \%: 1,00-1,89)$ e parto prematuro (OR $=2,98,95 \% \mathrm{Cl}: 2,48-3,58)$ em comparação ao grupo com idade $>20$ anos (HUANG CC, et al., 2015). Corroborando com este cenário, uma pesquisa realizada entre 2008 e 2009 no Brasil com 820 gestantes adolescentes (12 a 18 anos) evidenciou a predominância de parto prematuro neste grupo (PIMENTA AM, et al., 2012).

Vale salientar que a morbidade perinatal é cinco vezes maior para os fetos com RCIU. A prevenção, apesar das limitações pela dificuldade em determinar o agente causal, melhora a qualidade de vida desde 0 nascimento até a idade adulta. Gestantes com este fator de risco necessitam de maior atenção em relação ao crescimento fetal, medidas da altura uterina, e exames ultrassonográficos (NETO ARM, CÓRDOBA JCM e PERAÇOLI JC, 2011). Com relação à etnia, outro dado levantado por essa pesquisa constatou que mulheres com antecedentes obstétricos e que se declararam de cor parda/morena apresentaram maior frequência de mortes fetais e neonatais. Outra análise sobre o mesmo tema evidenciou que a cor preta e parda da mãe eleva o risco de óbitos neonatais, sinalizando que esses resultados são expressões de disparidades sociais que pode ser um fator limitante de acesso aos serviços de saúde (OLIVEIRA EFV, GAMA SGN e SILVA CMFP, 2010).

Ao associarmos o estado civil com as condições de doenças pré-existentes, mulheres separadas/divorciadas apresentaram resultados significativos para doenças hipertensivas e cirurgia bariátrica. Quando associada a antecedentes obstétricos, apresentou relevância para mais de três cesáreas. Porém, em alguns estudos, não se verificou relação significativa entre estado civil e essas variáveis (PÁDUA KS, et al., 2010; ASSIS TR, VIANA FP e RASSI S, 2008). Outra condição pré-clínica relevante encontrada em mulheres separadas/divorciadas foi a obesidade mórbida, entretanto, em outro estudo por investigação de inquérito domiciliar, no Maranhão, com 1005 adultos, sendo 612 mulheres, revelou por meio de regressão logística que não há associação estatística entre situação conjugal e obesidade em mulheres (VELOSO HJF e SILVA AAM, 2010). Já em mulheres solteiras, a condição pré-clínica prevalente foi a dependência de drogas lícitas/ilícitas, em concordância com outro estudo que, ao entrevistar 25 gestantes usuárias de drogas, verificou que $56 \%$ eram solteiras e relataram que além de se sentirem julgadas, não receberam apoio e nem acompanhamento adequado para o enfrentamento do problema (KASSADA DS, MARCON SS e WAIDMAN MAP, 2014).

Embora poucos estudos enfatizem a importância da relação estável e a presença do parceiro no contexto reprodutivo, sobretudo pelo suporte emocional (CARVALHO JBL, BRITO RS e SANTOS DLAS, 2011), ressalta-se que situação conjugal instável é mais um fator de risco na gestação e que pode gerar complicações obstétricas, tendo em vista a carência de afeto e a ausência do parceiro para compartilhar as responsabilidades sobre a vida do filho (REZENDE CL e SOUZA JC, 2012). 
Ao associar a escolaridade e as condições pré-existentes neste estudo, constatou-se que mulheres com $<8$ anos de estudo representam fator significativo para hipertensão arterial e epilepsia. Já em outra investigação, não houve relação entre escolaridade e síndromes hipertensivas (OLIVEIRA EFV, GAMA SGN e SILVA CMFP, 2010) e nem estudos que associassem a baixa escolaridade e a epilepsia. Porém, no que se refere à hipertensão arterial, uma análise sobre os níveis pressóricos em 94 gestantes constatou que a baixa escolaridade é um fator de risco para síndrome hipertensiva (GOMES AS, et al., 2013).

A gemelaridade como intercorrência clínica também se destacou em mulheres com baixa escolaridade (< 8 anos de estudos). No entanto, não foram encontrados estudos que evidenciassem esta associação. Os achados se deram apenas em uma pesquisa que relatou as várias teorias apresentadas para explicar os determinantes da gemelaridade, como a herança genética, fatores ambientais como idade materna, paridade, grupo étnico, antecedentes obstétricos, indução da ovulação, classe social, entre outros, porém em nenhum momento foi citada a baixa escolaridade (RAMOS HAC e CUMAN RKN, 2009).

Vale ressaltar que o Ministério da Saúde apresenta a variável baixa escolaridade como fator desfavorável à realização do pré-natal na gravidez, justificando a deficiência no entendimento das orientações dos profissionais de saúde, sendo este um fator de risco que deve ser acompanhado mais de perto (LINHARES JJ, et al., 2014).

Ao realizar o levantamento da variável religião, os resultados apontaram que as gestantes que declararam não ter religião apresentaram neoplasias como condições pré-existentes, hemorragias do segundo semestre como antecedentes obstétricos e toxoplasmose e amniorrexe prematura como intercorrências clínicas. Para as que se classificaram como evangélicas, observou-se amniorrexe prematura como intercorrência clínica.

Mediante esses resultados, não foram encontrados estudos que relacionassem religião com esses riscos gestacionais, entretanto, apenas um afirma que religião não interfere na qualidade de vida da gestante (REZENDE CL e SOUZA JC, 2012). Enquanto outro evidencia que o apelo religioso pode ser um fator protetor ao comportamento suicida, além de oferecer um apoio social, auto-estima e enfrentamento de problemas no período gestacional (BENUTE GRG, et al., 2011).

\section{CONCLUSÃO}

Identificaram-se associações entre o perfil sociodemográfico com condições clínicas pré-existentes, antecedentes obstétricos e intercorrências clínicas na gestação de alto risco, atribuindo aos ambulatórios especializados a necessidade de realizar ações para minimizar os desfechos desfavoráveis e melhorar a qualidade dos serviços. Além disso, os resultados apresentados poderão auxiliar na realização de protocolos de atendimento e assistência segmentada por parte da equipe de saúde.

\section{REFERÊNCIAS}

1. ASSIS TR, et al. Estudo dos principais fatores de risco maternos nas síndromes hipertensivas da gestação. Arq. Bras. Cardiol., 2008; 91(1):11-17.

2. BENUTE GRG, et al. Risco de suicídio em gestantes de alto risco: um estudo exploratório. Rev Assoc Med Bras. 2011; 57(5):583-587.

3. BRASIL. Ministério da Saúde. Gabinete do Ministro. Portaria no 1.459, de 24 de junho de 2011. Institui, no âmbito do Sistema Único de Saúde - SUS - a Rede Cegonha. Diário Oficial da União, Brasília, DF, 2011.

4. BRASIL. Ministério da Saúde. Secretaria de Atenção à Saúde. Departamento de Ações Programáticas Estratégicas. Gestação de alto risco: manual técnico. 5. ed. Brasília, DF, 2012a.

5. BRASIL. Ministério da Saúde. Secretaria de Atenção à Saúde. Departamento de Atenção Básica. Atenção ao prénatal de baixo risco. Brasília, DF, $2012 b$.

6. BROSH K, et al. Teratogenic determinants of first-trimester exposure to antiepileptic medications. J. Popul. Ther. Clin. Pharmacol., Canadá, 2011; 18(1):89-98.

7. CARVALHO JBL, et al. Percepção do homem sobre a atenção recebida dos profissionais que assistem a companheira com síndromes hipertensivas. Cienc. Cuid. Saude, 2011; 10(2):322-329. 
8. FRIDMAN M, et al. Trends in maternal morbidity before and during pregnancy in California. Am. J. Public Health, Oxford, 2014; 104(S1):S49-S57.

9. GOMES AS, et al. Análise dos níveis pressóricos em gestantes no diagnóstico precoce da síndrome hipertensiva gestacional. Rev. Eletr. Enf., 2013; 15(4):923-931.

10. GONÇALVES ZR, MONTEIRO DLM. Maternal complications in women with advanced maternal age. FEMINA, 2012; 40(5):275-279.

11. HUANG CC, et al. Comparison of medical issues in antenatal and perinatal periods in early youth, adolescent, and young adult mothers in Taiwan: a 10-year nationwide study. BMC Pregnancy Childbirth, Londres, 2014; 14(1):260.

12. KASSADA DS, et al. Percepção e práticas de gestantes atendidas na atenção primária frente ao uso de drogas. Esc. Anna Nery, 2014; 18(3):428-434.

13. LEITE GKC, et al. Miomectomia em gestação de segundo trimestre: relato de caso. Rev Bras Ginecol Obstet. 2010; 32(4):198- 201.

14. LINHARES JJ, et al. Fatores associados à via de parto em mulheres com pré-eclâmpsia. Rev Bras Ginecol Obstet. 2014; 36(6):259-263.

15. LISONKOVA S, et al. Does advanced maternal age confer a survival advantage to infants Born at early gestation? BMC Pregnancy Childbirth. 2013; 13(87).

16. LUNARDI LL, et al. Quality of life in pregnant women with epilepsy versus women with epilepsy. Arq Neuropsiquiatr $2011 ; 69(2-B): 336-341$

17. NAJAFI MR. et al. The course and outcome of pregnancy and neonatal situation in epileptic women. Adv. Biomed. Res., Mumbai, 2012; 1(1):4.

18. NETO ARM, et al. Etiologia da restrição de crescimento intrauterino (RCIU). Com. Cienc. Saude,, 2011; $22(1): 21-30$.

19. OLIVEIRA EFV, et al. Gravidez na adolescência e outros fatores de risco para mortalidade fetal e infantil no Município do Rio de Janeiro, Brasil. Cad. Saude Publica, 2010; 26(3):567-578.

20. OMS - Organização Mundial de Saúde. Declaração da OMS sobre taxas de cesáreas (Online). 2015.

21. OSAVA RH, et al. Caracterização das cesarianas em centro de parto normal. Rev. Saude Publica, São Paulo, v. 45, n. 6, p. 1036-1043, 2011.

22. PÁDUA KS, et al. Fatores associados à realização de cesariana em hospitais brasileiros. Rev. Saude Publica, 2010; 44(1):70.

23. PARANÁ. Secretaria Estadual de Saúde. Linha guia: mãe paranaense. Curitiba: SESA, 2018. p.63.

24. PIMENTA AM, et al. Programa "Casa das Gestantes": Perfil das usuárias e resultados da assistência à saúde materna e perinatal. Texto Contexto Enferm., 2012; 21(4):912-20.

25. RAMOS HAC, CUMAN RKN. Fatores de risco para prematuridade: pesquisa documental. Esc. Anna Nery, 2009; 13(2):297-304.

26. REIS LLAS, FERRARI R. Malformações congênitas: perfil sociodemográfico das mães e condições de gestação. Rev enferm UFPE., 2014; 8(1):98-106.

27. REIS LV, et al. Anomalias Congénitas Identificadas ao Nascimento em Recém-Nascidos de Mulheres Adolescentes. Acta Med Port. 2015; 28(6):708-714.

28. REZENDE CL, SOUZA JC. Qualidade de vida das gestantes de alto risco de um centro de atendimento à mulher. inFormação, 2012; 16(16):45-69.

29. VELOSO HJF, SILVA AAM. Prevalência e fatores associados à obesidade abdominal e ao excesso de peso em adultos maranhenses. Rev Bras Epidemiol. 2010; 13(3):400-412.

30. WHO - World Health Organization. World health statistics 2014. Geneva, 2014. 